\section{Atividade física em trabalhadores de Centros de Atenção Psicossocial do Sul do Brasil: tendências temporais}

\author{
Physical activity in staff workers at Centers for \\ Psychosocial Care in southern Brazil: temporal \\ trends
}

\author{
Actividad física de los trabajadores en los \\ Centros de Atención Psicosocial del sur de Brasil: \\ tendencias temporales
}

Programa de Pós-graduação
em Educação Física,
Universidade Federal de
Pelotas, Brasil.
2 Núcleo de Educação,
Avaliação e Produção
Pedagógica em Saúde,
Universidade Federal do Rio
Grande do Sul, Porto Alegre,
Brasil.
3 Programa de Pós-
graduação em Enfermagem,
Universidade Federal de
Pelotas, Pelotas, Brasil.
Correspondência
J. S. Jerônimo
Programa de Pós-graduação
em Educação Física,
Universidade Federal de
Pelotas.
Rua Luís de Camões 625,
Pelotas, RS 96055-630,
Brasil.
jefersonsj@yahoo.com.br

Abstract

The aim of the study was to analyze temporal trends of physical activity among staff workers in Centers for Psychosocial Care and associated factors in southern Brazil from 2006 to 2011. This cross-sectional study was part of the Evaluation of Centers for Psychosocial Care in Southern Brazil/CAPSUL. Physical and mental health variables were collected using the Self-Report Questionnaire (SRQ-20), and physical activity was measured with the International Physical Activity Questionnaire (IPAQ). Participation included 435 staff workers in 2006 and 546 in 2011. Total prevalence rates were: physical activity $(\geq 150$ minutes/week) $23.2 \%$ in 2006 and $17.6 \%$ in 2011 and minor psychiatric disorders $11 \%$ and $8.4 \%$. There was no statistically significant difference in physical activity between men and women. In 2006, individuals with less schooling $(p=0.03)$ and lower income $(p=0.01)$ showed higher levels of physical activity. In 2011, staff workers in larger cities showed higher levels of physical activity $(p=0.02)$. Interventions are needed to promote physical activity in this population, especially among staff workers at Centers for Psychosocial Care in smaller municipalities.

Motor Activity; Mental Health Services; Occupational Health; Mental Health; Health Services
Jeferson Santos Jerônimo 1,2 Vanda Maria da Rosa Jardim 3 Luciane Prado Kantorski 3 Marlos Rodrigues Domingues 1

\section{Resumen}

O objetivo foi apresentar tendências temporais de atividade física e fatores associados em trabalhadores de Centros de Atenção Psicossocial (CAPS) da Região Sul do Brasil entre 2006 e 2011. Pesquisa transversal, parte do estudo Avaliação dos CAPS da Região Sul do Brasil/CAPSUL. Foram coletadas variáveis de saúde física, saúde mental por meio do Self-Report Questionnaire (SRQ-20) e atividade física usando-se o International Physical Activity Questionnaire (IPAQ). Participaram 435 trabalhadores de CAPS em 2006 e 546 trabalhadores em 2011. As prevalências totais de atividade física ( $\geq 150$ minutos semanais) foram $23,2 \% \mathrm{em}$ 2006 e 17,6\% em 2011, e de distúrbios psiquiátricos menores $11 \%$ e 8,4\%. Não houve diferença na atividade física de homens e mulheres. Em 2006, sujeitos com menor escolaridade $(p=0,03)$ e menor renda $(p=0,01)$ apresentaram maior nivel de atividade física. Em 2011, trabalhadores de CAPS localizados em municípios de grande porte apresentaram maior nível de atividade física ( $p=$ 0,02). São necessárias intervenções promotoras de atividade física nessa população, principalmente em trabalhadores de CAPS residentes em municípios de pequeno porte.

Atividade Motora; Serviços de Saúde Mental; Saúde do Trabalhador; Saúde Mental; Serviços de Saúde 


\section{Introdução}

A literatura tem demonstrado os benefícios da atividade física para a saúde física e mental; para a melhora da aptidão cardiorrespiratória; muscular; para a saúde óssea e função cognitiva 1. Além de promover melhor qualidade de vida no ambiente de trabalho 2 , trabalhadores com níveis de atividade física mais elevados apresentam menor peso corporal, maior consumo de frutas e vegetais, menor consumo de tabaco e melhor bem-estar mental ${ }^{3}$. Nesse sentido, a área da atividade física e saúde vem pesquisando outras temáticas em saúde pública como a saúde do trabalhador 4,5,6.

No Brasil, nos últimos anos, observa-se um aumento na força de trabalho no setor da saúde, entretanto, deste processo decorrem disputas orçamentárias gerando falta de condições de trabalho e remuneração inadequada 7,8 , o que pode afetar negativamente o nível de atividade física e outras condições de saúde desses trabalhadores ${ }^{9}$. O tema saúde do trabalhador é pesquisado com mais força no meio acadêmico, principalmente a partir da década de 1990, e atualmente preocupa-se com a saúde do trabalhador de serviços de saúde 10,11, entre estes os Centros de Atenção Psicossocial (CAPS) 12.

Esse serviço tornou-se política pública em 2001 com a Lei no 10.216, e desde então vem crescendo ano a ano. Atualmente no Brasil existem 1.742 CAPS, a Região Sul conta com 323 unidades, atingindo uma cobertura de 0,91 unidade por 100 mil habitantes, o que é considerado pelo Ministério da Saúde do Brasil como uma cobertura muito boa, por estar acima de 0,70 unidade por 100 mil habitantes 13,14.

A literatura demonstra que trabalhadores da atenção básica no Brasil são acometidos por diversos problemas como tabagismo, insatisfação com a saúde, consumo regular de medicamentos e distúrbios psiquiátricos menores 15,16 . Por outro lado, apresentam elevadas prevalências de atividade física, considerando o conjunto das atividades físicas realizadas no tempo de lazer, deslocamento, serviços domésticos e, principalmente, atividades ocupacionais. Do total da amostra de trabalhadores de unidades básicas de saúde (UBS) das regiões Sul e Nordeste do Brasil, $72,5 \%$ são ativos fisicamente 17 .

Referente à saúde do trabalhador de CAPS, embora se observe um aumento na produção científica relativa ao tema, a maioria dos estudos é com abordagens metodológicas qualitativas ou com amostras reduzidas em estudos transversais de pouca validade externa ${ }^{12}$. Além disso, até o momento, não foram encontrados estudos que avaliem atividade física nessa população.
Nosso estudo comparou duas pesquisas transversais com amostras representativas da Região Sul do Brasil, com o objetivo de demonstrar tendências temporais de atividade física e os fatores associados em trabalhadores de CAPS desta região do país entre 2006 e 2011.

\section{Métodos}

Pesquisa epidemiológica, observacional, transversal com coletas em dois pontos temporais. Parte do estudo Avaliação dos Centros de Atenção Psicossocial da Região Sul do Brasil, conhecido como CAPSUL 18 , resultante de uma demanda do Ministério da Saúde do Brasil. Foram coletados dados da população de trabalhadores de CAPS da Região Sul do Brasil em 2006 e 2011.

O cálculo amostral foi realizado no programa Epi Info 6.04 (Centers for Disease Control and Prevention, Atlanta, Estados Unidos) com valor de alfa de $5 \%$ e poder de $95 \%$, e embasou-se em características das unidades de CAPS da Região Sul do Brasil e dos trabalhadores destas unidades. Com referência às unidades de CAPS, o cálculo foi estruturado por múltiplos estágios e considerou: oferta de serviços nos três estados da região; concentração populacional; distribuição geográfica e modelo de CAPS, chegando a trinta unidades de CAPS I e II, uma em cada município, selecionadas aleatoriamente para 2006: três no Paraná, nove em Santa Catarina e dezoito no Rio Grande do Sul. Para 2011 foram selecionadas, com o mesmo procedimento, quarenta unidades de CAPS I, II e III: 12 no Paraná, dez em Santa Catarina e 18 no Rio Grande do Sul.

Em relação aos trabalhadores, o cálculo levou em conta uma estimativa de 15 profissionais por unidade, portanto, multiplicando-se 15 pelo número de unidades em cada estado da Região Sul para cada coleta (2006 e 2011), obtivemos um “N" estimado de 450 trabalhadores em 2006 e de 600 em 2011. Foi utilizado como critério de inclusão estar trabalhando na unidade selecionada em qualquer cargo ou função, sob qualquer tipo de contrato, inclusive contrato de estágio.

Todos os trabalhadores de cada unidade selecionada foram convidados a participar do estudo, os instrumentos foram autoaplicados com supervisão do entrevistador para diminuir eventuais dúvidas, as variáveis foram coletadas de forma contínua e categorizadas durante as análises. Para este trabalho utilizou-se as variáveis demográficas: sexo (masculino e feminino); idade (16-29 anos, 30-39, 40-49 e $\geq 50$ anos); cor da pele (branca e não branca); peso e estatura para cálculo do índice de massa corporal (IMC) 19 . Socioeconômicas: escolaridade (1-8 anos, 9-12 e 
$\geq 13$ anos); possuir pós-graduação (não e sim); nível profissional (superior, técnico e outros); renda individual (quartis, $25 \%$ em cada categoria); ter companheiro (não e sim). Ocupacionais: tipo de CAPS (I, II e III, de acordo com a Portaria no 336/GM 20, CAPS I para municípios de $20 \mathrm{mil}$ a 70 mil habitantes, CAPS II de 70 mil a $200 \mathrm{mil}$ e CAPS III mais de 200 mil habitantes); tempo de trabalho no CAPS (até 1 ano, até 2 , até 5 e $\geq 6$ anos); carga horária semanal (até 20 horas e 21 a 40 horas). Saúde: tabagismo (não e sim) IMC 19 (normal, sobrepeso e obeso); doenças crônicas não transmissíveis (não e sim) e distúrbios psiquiátricos menores (não e sim, mensurados pelo Self-Report Questionnaire-SRQ-20).

O SRQ-20 é um instrumento recomendado pela Organização Mundial da Saúde (OMS) para identificar distúrbios psiquiátricos menores 21, foi validado para a população brasileira por Mari \& Williams 22, composto por vinte questões que avaliam quatro grupos de sintomas de distúrbios psiquiátricos menores: comportamento ansioso e depressivo; decréscimo de energia; sintomas somáticos e humor depressivo. Pode ser utilizado em diferentes populações e demonstra bom desempenho para rastrear distúrbios psiquiátricos menores em ambientes de trabalho 23 . Apresenta bom desempenho para discriminação de casos e não casos e efetividade para o uso em larga escala, comparado a outros instrumentos, características que o indicam para estudos de base populacional 24. Foram utilizados pontos de corte $\geq 6$ questões positivas para homens (sensibilidade de $89 \%$ e especificidade de $91 \%$ ) e $\geq 7$ para mulheres (sensibilidade de $86 \%$ e especificidade de $77 \%$ ), conforme indica o estudo de validação para a população brasileira 22 .

Considerou-se como desfecho o nível de atividade física, dicotomizado em não e sim, mensurado pelo International Physical Activity Questionnaire - Short Form (IPAQ-curto), instrumento recomendado pela OMS e validado internacionalmente por Craig et al. 25, e para a população brasileira por Matsudo et al. 26. Esse instrumento mede simultaneamente atividades físicas realizadas no tempo livre ou de lazer, deslocamento, atividades domésticas e ocupacionais. Apresenta resultados satisfatórios e compatíveis com outros instrumentos utilizados internacionalmente, não apresenta diferenças entre as formas de aplicação por telefone e autoaplicável, apresenta praticidade e rapidez na aplicação, o que possibilita coletar informações de grandes grupos populacionais, além de ser indicado para estudos de acompanhamento 26 . Foi utilizado o ponto de corte de 150 minutos semanais de atividade física para classificação dos sujeitos como ativos, conforme a recomendação da OMS 27, somando-se os minutos de caminhada, de atividades de intensidade moderada e vigorosa.

Nas análises estatísticas foram utilizados os testes qui-quadrado de Pearson para as variáveis dicotômicas e qui-quadrado de tendência linear para as variáveis politômicas ordinais. Foram feitos modelos de regressão de Poisson para controlar possíveis fatores de confusão 28 , um para cada coleta: 2006 e 2011. O modelo de regressão de Poisson é indicado para desfechos que apresentam prevalências maiores do que $10 \%$, os modelos utilizados foram compostos por cinco níveis hierárquicos: 1o: sexo, idade e cor da pele; 2o: ter companheiro, escolaridade, possuir pós-graduação, nível profissional e renda; 3o: tipo de CAPS (I, II e III), tempo de trabalho no CAPS, carga horária semanal, turno de trabalho; 4o: tabagismo, IMC, doenças crônicas não transmissíveis, distúrbios psiquiátricos menores; e $5^{\circ}$ nível: desfecho atividade física. Foram incluídas nos modelos de regressão aquelas variáveis que apresentaram valor de $\mathrm{p}<0,2$ durante as análises brutas, conforme indica a literatura sobre estatística para a área da saúde ${ }^{28}$. As análises foram feitas no pacote estatístico Stata 12.1 (Stata Corp., College Station Estados Unidos).

As coletas de dados, 2006 e 2011, foram aprovadas pelos Comitês de Ética em Pesquisa envolvendo seres humanos da Faculdade de Medicina da Universidade Federal de Pelotas (UFPel; protocolo no 074/2005) e da Faculdade de Enfermagem da UFPel (protocolo no 176/2011), respectivamente, e todos os sujeitos assinaram o Termo de Consentimento Livre e Esclarecido antes das coletas.

\section{Resultados}

Em 2006 foram atingidos 96,6\% do cálculo amostral $(\mathrm{N}=435)$. A prevalência total de ativos na amostra foi de 23,2\% com mediana de 75 minutos semanais. Na coleta de 2011 atingiu-se $91 \%$ ( $\mathrm{N}=546)$ e a prevalência ativos foi de $17,6 \% \mathrm{com}$ mediana de 70 minutos semanais. Os pequenos porcentuais de perdas foram em razão de profissionais que se encontravam de férias ou de licença durante os períodos das coletas. Em ambas as coletas (2006 e 2011), observamos maior prevalência de mulheres, com média de idade de 37,4 $( \pm 10,3)$ anos e 37,5 ( $\pm 10,8)$ anos; de cor da pele branca; com companheiro; sem pós-graduação; de nível profissional superior; com mediana de renda individual de $\mathrm{R} \$ 1.050,00( \pm \mathrm{R} \$ 888,00) \mathrm{em}$ 2006 e de $\mathrm{R} \$ 1.200,00$ ( $\pm \mathrm{R} \$ 1.347,00)$ em 2011; com carga horária de 21 e 40 horas semanais; que trabalhavam no turno do dia. As prevalências totais de distúrbios psiquiátricos menores 
foram $11 \%$ em 2006 e $8,4 \%$ em 2011 , sendo maior em mulheres $(12,4 \%$ e $9,2 \%)$ do que em homens (6,2\% e 5,5\%). A Tabela 1 apresenta um comparativo entre as prevalências totais de atividade física e das variáveis independentes das amostras de 2006 e 2011, demonstrando as principais tendências temporais.

Nas análises bivariadas, em 2006 não houve diferença entre homens e mulheres em relação à atividade física. Observou-se associação inversa entre nível profissional ( $p=0,02$ ), renda individual ( $p=0,005)$ e atividade física, ou seja, quanto maior o nível profissional e de renda menor o nível de atividade física. Em 2011, mulheres foram ligeiramente mais ativas fisicamente do que homens ( $p=0,05)$; observou-se tendência positiva entre renda individual $(p=0,03)$, tempo de trabalho no CAPS ( $\mathrm{p}=0,003$ ) e atividade física, isto é, quanto maior a renda, com exceção do quartil mais alto, e maior o número de anos de trabalho no CAPS, maior o nível de atividade física. Além disso, tipo de CAPS apresentou significância limítrofe com atividade física $(p=0,05)$ : trabalhadores de CAPS tipo III foram mais ativos fisicamente. A Tabela 2 apresenta o comparativo entre as prevalências de atividade física conforme as variáveis independentes das amostras de 2006 e 2011.

Tabela 1

Prevalência de atividade física e das variáveis independentes das amostras de trabalhadores de Centros de Atenção Psicossocial (CAPS). Região Sul do Brasil, 2006 e 2011.

\begin{tabular}{|c|c|c|}
\hline Variáveis & $2006(N=435)$ & $2011(N=546)$ \\
\hline & $\%$ & $\%$ \\
\hline \multicolumn{3}{|l|}{ Atividade física } \\
\hline Não & 76,8 & 82,4 \\
\hline Sim & 23,2 & 17,6 \\
\hline \multicolumn{3}{|l|}{ Sexo } \\
\hline Masculino & 22,3 & 20,1 \\
\hline Feminino & 77,7 & 79,9 \\
\hline \multicolumn{3}{|l|}{ Idade (anos) } \\
\hline $16-29$ & 30,0 & 32,7 \\
\hline $30-39$ & 30,5 & 26,6 \\
\hline $40-49$ & 28,4 & 27,9 \\
\hline$\geq 50$ & 11,1 & 12,7 \\
\hline \multicolumn{3}{|l|}{ Cor da pele } \\
\hline Branca & 87,7 & 83,4 \\
\hline Não branca & 12,3 & 16,6 \\
\hline \multicolumn{3}{|l|}{ Companheiro } \\
\hline Não & 48,2 & 47,2 \\
\hline Sim & 51,8 & 52,8 \\
\hline \multicolumn{3}{|c|}{ Escolaridade (anos de estudo) } \\
\hline $1-8$ & 14,6 & 16,5 \\
\hline $9-12$ & 22,6 & 25,5 \\
\hline$\geq 13$ & 62,8 & 57,9 \\
\hline \multicolumn{3}{|l|}{ Pós-graduação } \\
\hline Não & 60,3 & 63,5 \\
\hline Sim & 39,7 & 36,5 \\
\hline \multicolumn{3}{|c|}{ Nível profissional } \\
\hline Superior & 54,4 & 54,6 \\
\hline Técnico & 17,5 & 20,8 \\
\hline Outros & 28,1 & 24,6 \\
\hline
\end{tabular}

(continua) 
Tabela 1 (continuação)

\begin{tabular}{|c|c|c|}
\hline Variáveis & $2006(N=435)$ & $2011(N=546)$ \\
\hline & $\%$ & $\%$ \\
\hline \multicolumn{3}{|l|}{ Tipo de CAPS } \\
\hline I & 44,1 & 47,2 \\
\hline$\|$ & 55,9 & 33,2 \\
\hline III & - & 19,6 \\
\hline \multicolumn{3}{|c|}{ Tempo de trabalho no CAPS (anos) } \\
\hline Até 1 & 29,9 & 37,8 \\
\hline Até 2 & 21,1 & 15,0 \\
\hline Até 5 & 31,1 & 26,6 \\
\hline$\geq 6$ & 17,8 & 20,6 \\
\hline \multicolumn{3}{|c|}{ Carga horária semanal (horas) } \\
\hline Até 20 & 32,1 & 30,6 \\
\hline $21-40$ & 67,9 & 69,4 \\
\hline \multicolumn{3}{|c|}{ Turno de trabalho } \\
\hline Dia & 95,8 & 93,7 \\
\hline Noite & 4,2 & 6,3 \\
\hline \multicolumn{3}{|l|}{ Tabagismo } \\
\hline Não & 82,2 & 89,3 \\
\hline Sim & 17,8 & 10,7 \\
\hline \multicolumn{3}{|l|}{ IMC } \\
\hline Normal & 61,7 & 56,8 \\
\hline Sobrepeso & 31,0 & 29,4 \\
\hline Obeso & 7,3 & 13,8 \\
\hline \multicolumn{3}{|c|}{ Doenças crônicas não transmissíveis } \\
\hline Não & 79,3 & 73,1 \\
\hline Sim & 20,7 & 26,9 \\
\hline \multicolumn{3}{|c|}{ Distúrbios psiquiátricos menores } \\
\hline Não & 89,0 & 91,6 \\
\hline $\operatorname{Sim}$ & 11,0 & 8,4 \\
\hline
\end{tabular}

IMC: índice de massa corporal.

Nas análises ajustadas, em 2006, escolaridade e renda individual apresentaram associação inversa com atividade física: sujeitos com menor escolaridade ( $p=003)$ e menor renda individual $(\mathrm{p}=0,01)$ apresentaram mais chances de serem ativos fisicamente, sendo que trabalhadores do maior quartil de renda apresentaram $50 \%$ a mais de chances de serem inativos fisicamente do que trabalhadores do menor quartil. Em 2011, trabalhadores de CAPS tipo III apresentaram 83\% a mais de chances de serem ativos em relação aos trabalhadores de CAPS tipo I ( $p=0,02)$. As Tabelas 3 e 4 apresentam as variáveis que compuseram os modelos de análise bruta e ajustada para 2006 e 2011, respectivamente.

\section{Discussão}

Foi feito um comparativo entre dois estudos transversais (2006 e 2011) relativos à população de trabalhadores de CAPS da Região Sul do Brasil para avaliar tendências temporais de atividade física e fatores associados.

Nosso trabalho observou diminuição na prevalência total de atividade física de 5,6\% entre 2006 e 20111, o que é preocupante no que se refere à saúde da população estudada, considerando as evidências científicas sobre os benefícios da prática regular de atividade física para a saúde física e mental 1 . A literatura tem demonstrado a diminuição na proporção de sujeitos ativos em diferentes populações, fenômeno decorrente do desenvolvimento de tecnologias nos ambientes laborais, domésticos e de transporte, gerando menor gasto energético para a manutenção da vida 29 , o que é benéfico para o avanço das socie- 
Tabela 2

Prevalência da atividade física conforme variáveis independentes da amostra de trabalhadores de Centros de Atenção

Psicossocial (CAPS). Região Sul do Brasil, 2006 e 2011.

\begin{tabular}{|c|c|c|c|c|}
\hline \multirow[b]{2}{*}{ Variáveis } & \multicolumn{2}{|c|}{$2006(N=435)$} & \multicolumn{2}{|c|}{$2011(N=546)$} \\
\hline & $\%$ & Valor de $p$ & $\%$ & Valor de $p$ \\
\hline Sexo & & 0,34 * & & 0,05 * \\
\hline Masculino & 26,8 & & 11,0 & \\
\hline Feminino & 22,2 & & 19,1 & \\
\hline Idade (anos) & & 0,99 ** & & 0,68 ** \\
\hline $16-29$ & 22,3 & & 16,4 & \\
\hline $30-39$ & 24,2 & & 16,0 & \\
\hline $40-49$ & 23,6 & & 19,2 & \\
\hline$\geq 50$ & 22,9 & & 21,7 & \\
\hline Cor da pele & & 0,20 * & & 0,25 \\
\hline Branca & 22,2 & & 18,6 & \\
\hline Não branca & 30,2 & & 13,5 & \\
\hline Companheiro & & 0,76 * & & 0,93 * \\
\hline Não & 23,9 & & 17,2 & \\
\hline Sim & 22,7 & & 17,5 & \\
\hline Escolaridade (anos de estudo) & & 0,06 ** & & 0,42 ** \\
\hline $1-8$ & 30,5 & & 13,9 & \\
\hline $9-12$ & 29,7 & & 21,3 & \\
\hline$\geq 13$ & 19,8 & & 18,0 & \\
\hline Pós-graduação & & 0,18 * & & 0,82 * \\
\hline Não & 25,6 & & 17,7 & \\
\hline Sim & 20,0 & & 17,1 & \\
\hline Nível profissional & & 0,02 ** & & 0,33 ** \\
\hline Superior & 18,0 & & 15,5 & \\
\hline Técnico & 29,3 & & 21,2 & \\
\hline Outros & 30,0 & & 19,4 & \\
\hline Renda individual (quartis) & & 0,005 ** & & 0,03 ** \\
\hline 1 (menor) & 35,7 & & 16,1 & \\
\hline 2 & 25,5 & & 21,6 & \\
\hline 3 & 16,5 & & 25,4 & \\
\hline 4 (maior) & 17,5 & & 11,4 & \\
\hline Tipo de CAPS & & 0,57 ** & & 0,05 ** \\
\hline I & 22,0 & & 14,7 & \\
\hline$\|$ & 24,3 & & 17,1 & \\
\hline III & - & & 25,2 & \\
\hline $\begin{array}{l}\text { Tempo de trabalho no CAPS } \\
\text { (anos) }\end{array}$ & & 0,88 ** & & 0,003 ** \\
\hline Até 1 & 24,6 & & 14,3 & \\
\hline Até 2 & 24,7 & & 32,0 & \\
\hline Até 5 & 23,7 & & 13,8 & \\
\hline$\geq 6$ & 20,0 & & 16,8 & \\
\hline Carga horária semanal (horas) & & 0,23 * & & 0,31 * \\
\hline Até 20 & 19,3 & & 15,0 & \\
\hline $21-40$ & 24,5 & & 18,6 & \\
\hline Turno de trabalho & & $0,91 *$ & & 0,34 * \\
\hline Dia & 23,4 & & 17,1 & \\
\hline Noite & 22,2 & & 23,5 & \\
\hline
\end{tabular}

(continua) 
Tabela 2 (continuação)

\begin{tabular}{|c|c|c|c|c|}
\hline \multirow[b]{2}{*}{ Variáveis } & \multicolumn{2}{|c|}{$2006(N=435)$} & \multicolumn{2}{|c|}{$2011(N=546)$} \\
\hline & $\%$ & Valor de $p$ & $\%$ & Valor de $p$ \\
\hline Tabagismo & & 0,37 * & & 0,04 * \\
\hline Não & 22,8 & & 16,5 & \\
\hline Sim & 27,6 & & 27,6 & \\
\hline IMC & & 0,87 ** & & 0,44 ** \\
\hline Normal & 22,9 & & 19,9 & \\
\hline Sobrepeso & 24,4 & & 15,0 & \\
\hline Obeso & 20,0 & & 18,0 & \\
\hline $\begin{array}{l}\text { Doenças crônicas não } \\
\text { transmissiveis }\end{array}$ & & 0,52 * & & 0,42 * \\
\hline Não & 22,8 & & 16,8 & \\
\hline Sim & 26,1 & & 19,7 & \\
\hline $\begin{array}{l}\text { Distúrbios psiquiátricos } \\
\text { menores }\end{array}$ & & 0,08 * & & 0,71 * \\
\hline Não & 22,0 & & 17,4 & \\
\hline Sim & 33,3 & & 19,6 & \\
\hline
\end{tabular}

IMC: índice de massa corporal.

* Qui-quadrado de Pearson:

** Qui-quadrado de tendência linear.

Tabela 3

Prevalência de atividade física, análises bruta e ajustada da amostra de trabalhadores de Centros de Atenção Psicossocial (CAPS). Região Sul do Brasil, 2006 ( $N=435)$.

\begin{tabular}{|c|c|c|c|c|c|}
\hline \multirow[t]{2}{*}{ Variáveis } & \multirow[t]{2}{*}{$\%$} & \multicolumn{2}{|c|}{ Análise bruta } & \multicolumn{2}{|c|}{ Análise ajustada * } \\
\hline & & RP (IC95\%) & Valor de $p$ & RP (IC95\%) & Valor de $p$ \\
\hline Cor da pele & & & 0,18 & & 0,18 \\
\hline Branca & 22,2 & 1,00 & & 1,00 & \\
\hline Não branca & 30,2 & $1,35(0,86-2,13)$ & & $1,05(0,66-1,75)$ & \\
\hline Escolaridade (anos de estudo) & & & 0,02 & & 0,03 \\
\hline $1-8$ & 30,5 & 1,00 & & 1,00 & \\
\hline 9-12 & 29,7 & $0,97(0,59-1,60)$ & & $0,99(0,59-1,66)$ & \\
\hline$\geq 13$ & 19,8 & $0,64(0,40-1,02)$ & & $0,94(0,54-1,64)$ & \\
\hline Pós-graduação & & & 1,88 & & 0,95 \\
\hline Não & 25,6 & 1,00 & & 1,00 & \\
\hline Sim & 20,0 & $0,78(0,54-1,12)$ & & $1,01(0,64-1,57)$ & \\
\hline Nível profissional & & & 0,006 & & 0,23 \\
\hline Superior & 18,0 & 1,00 & & 1,00 & \\
\hline Técnico & 29,3 & $1,62(1,04-2,54)$ & & $1,37(0,80-2,35)$ & \\
\hline Outros & 30,0 & $1,66(1,12-2,45)$ & & $1,38(0,81-2,36)$ & \\
\hline Renda individual (quartis) & & & 0,002 & & 0,01 \\
\hline 1 (menor) & 35,7 & 1,00 & & 1,00 & \\
\hline 2 & 25,5 & $0,71(0,46-1,09)$ & & $0,68(0,42-1,09)$ & \\
\hline 3 & 16,5 & $0,46(0,27-0,77)$ & & $0,49(0,26-0,94)$ & \\
\hline 4 (maior) & 17,5 & $0,49(0,29-0,81)$ & & $0,50(0,27-0,92)$ & \\
\hline Distúrbios psiquiátricos menores & & & 0,06 & & 0,22 \\
\hline Não & 22,0 & 1,00 & & 1,00 & \\
\hline Sim & 33,3 & $1,51(0,97-2,36)$ & & $1,34(0,83-2,16)$ & \\
\hline
\end{tabular}

IC95\%: intervalo de 95\% de confiança; RP: razão de prevalência.

* Regressão de Poisson. 
Prevalência de atividade física, análises bruta e ajustada da amostra de trabalhadores de Centros de Atenção Psicossocial (CAPS). Região Sul do Brasil, 2011 ( $N=546)$.

\begin{tabular}{|c|c|c|c|c|c|}
\hline \multirow[t]{2}{*}{ Variáveis } & \multirow[t]{2}{*}{$\%$} & \multicolumn{2}{|c|}{ Análise bruta } & \multicolumn{2}{|c|}{ Análise ajustada * } \\
\hline & & RP (IC95\%) & Valor de $p$ & RP (IC95\%) & Valor de $\mathrm{p}$ \\
\hline Sexo & & & 0,06 & & 0,06 \\
\hline Masculino & 11,0 & 1,00 & & 1,00 & \\
\hline Feminino & 19,1 & $1,73(0,98-3,06)$ & & $1.79(1,03-3,10)$ & \\
\hline Tipo de CAPS & & & 0,02 & & 0,02 \\
\hline I & 14,7 & 1,00 & & 1,00 & \\
\hline II & 17,1 & $1,16(0,75-1,79)$ & & $1,17(0,75-1,82)$ & \\
\hline III & 25,2 & $1,71(1,10-2,65)$ & & $1,83(1,18-2,82)$ & \\
\hline Tabagismo & & & 0,03 & & 0,01 \\
\hline Não & 16,5 & 1,00 & & 1,00 & \\
\hline Sim & 27,6 & $1,67(1,05-2,65)$ & & $1,78(1,12-2,81)$ & \\
\hline
\end{tabular}

IC95\%: intervalo de 95\% de confiança; RP: razão de prevalência.

* Regressão de Poisson.

dades, mas prejudicial para a saúde das populações ${ }^{30}$. Contudo, as prevalências totais observadas em nosso estudo (23,2\% em 2006 e 17,6\% em 2011), além de apresentarem um declínio, são menores do que as relatadas na literatura, tanto na população em geral do Brasil e da Região Sul do país como na população de trabalhadores da saúde.

Nesse sentido, o estudo Vigilância de Fatores de Risco e Proteção para Doenças Crônicas por Inquérito Telefônico (VIGITEL) 31 demonstrou que $85,1 \%$ da população brasileira são ativos fisicamente em pelo menos um dos domínios (tempo livre, deslocamento, trabalho e ambiente doméstico) de avaliação da atividade física, resultado três vezes maior do que o observado em 2006 e cinco vezes maior do que o de 2011, sendo que as mulheres apresentaram uma prevalência um pouco maior $(85,4 \%)$ do que os homens $(84,8 \%)$, tendência semelhante à observada em 2011: mulheres $(19,1 \%)$ e homens (11\%), com significância limítrofe $(p=0,05)$. Além disso, o VIGITEL 31 demonstrou aumento significativo na prevalência total de atividade física no tempo livre, o que também ocorreu na população masculina, resultados contrários aos nossos que demonstraram diminuição na prevalência total de atividade física e a inversão na categoria de gênero mais prevalente.

Embora o VIGITEL 31 apresente prevalências de atividade física até cinco vezes maiores do que as que encontramos, cabe resaltar que se trata de uma pesquisa realizada somente nas capitais do país, ou seja, em cidades de grande porte, o que não é a realidade da maioria dos municípios brasileiros e, além disto, o instrumento utilizado foi o IPAQ-forma longa aplicado por telefone. Tais aspectos dificultam as comparações com os nossos achados, por outro lado, é uma pesquisa que abrange todo o território nacional, fornece dados que podem ser relativizados à população brasileira.

Estudo de Hallal et al. ${ }^{29}$ que compilou o nível de inatividade física em 122 países, demonstrou que no Brasil 50,8\% da população com 15 anos ou mais de idade são ativos fisicamente, número duas vezes maior do que o encontrado em $2006(23,2 \%)$ e três vezes maior do que o de 2011 (17,6\%). O mesmo estudo ainda demonstrou que no Brasil os homens $(52,8 \%)$ são mais ativos fisicamente do que as mulheres $(48,4 \%)$, achado semelhante ao da amostra de 2006: homens $(26,8 \%)$ e mulheres $(22,2 \%)(p=0,34)$.

Estudos de base populacional realizados na região Sul do Brasil que também utilizaram o IPAQ-curto 32,33 na população adulta, demonstraram prevalências de atividade física $(61 \% \mathrm{e}$ $70,4 \%$ ) de 2 a 3 vezes maiores do que os nossos achados de $2006(23,2 \%)$ e de 3 a 4 vezes maiores do que os de 2011 (17,6\%). Outros resultados discordantes são as medianas de 260 e 240 minutos por semana de atividade física, contrastando com os nossos resultados que demonstraram medianas de 75 minutos semanais de atividade física em 2006 e de 70 minutos semanais em 2011. Por outro lado, um resultado semelhante foi a associação inversa entre renda e atividade física, pois sujeitos com menor renda foram 
mais ativos fisicamente, o que também foi evidenciado na amostra de 2006, tanto na análise bruta $(\mathrm{p}=0,002)$ quanto na ajustada $(\mathrm{p}=0,01)$. Em contraposição, em 2011, nas análises bivariadas observamos o contrário, sujeitos com maior renda foram mais ativos fisicamente $(p=0,03$ ), entretanto, a associação se perdeu nas análises ajustadas.

Outro estudo realizado na Região Sul que também utilizou o IPAQ-curto em adultos residentes em áreas de abrangência de UBS 34, demonstrou que $76 \%$ praticavam atividade física, sendo que $61 \%$ afirmaram que a atividade física era benéfica à saúde e $76,1 \%$ disseram que tinham recebido prescrição de atividade física na UBS no último ano. Talvez a orientação recebida por meio da prescrição tenha influenciado na proporção de atividade física neste estudo, o que dificulta a comparação com os nossos achados, contudo, os números são mais elevados.

Em relação a trabalhadores de outros serviços de saúde, um estudo realizado nas regiões Sul e Nordeste do Brasil que também utilizou o IPAQ-curto 17 demonstrou que $71,8 \%$ dos trabalhadores de UBS da Região Sul praticavam atividade física, o que é três vezes maior do que a prevalência encontrada na coleta de 2006 e quatro vezes maior do que a de 2011. Porém, uma semelhança à coleta de 2011 foi a maior prevalência de mulheres ativas fisicamente $(19,1 \%)$, quando comparadas aos homens (11\%).

Ainda em relação à população do Sul do Brasil, estudo realizado por Siqueira et al. $35 \mathrm{com}$ 12.402 indivíduos em cem cidades do país, demonstrou que no Brasil $17,4 \%$ dos adultos praticam atividade física no tempo livre ou de lazer, resultado semelhante ao que encontramos na amostra de 2011 (17,6\%). Na Região Sul, Siqueira et al. 35 observaram valor idêntico $(17,6 \%)$ ao que encontramos em 2011, sugerindo que os trabalhadores de CAPS do Sul do país analisados em nosso estudo talvez tenham relatado a atividade física de lazer, já que em ambas as coletas a maior parte dos sujeitos apresentou carga horária semanal de 21 a 40 horas (67,9\% em 2006 e 69,4\% em 2011). Outro resultado semelhante ao nosso foi a relação inversa entre atividade física e escolaridade $(p<0,001)$ apresentada por Siqueira et al. 35. Observamos a mesma relação em 2006, na análise ajustada sujeitos com maior escolaridade apresentaram menor nível de atividade física $(\mathrm{p}=0,02)$.

Embora não tenha apresentado associação com atividade física, observamos aumento na prevalência de obesidade de 7,3\% em 2006 para $13,8 \%$ em 2011, e aumento na proporção de doenças crônicas não transmissíveis de $20,7 \%$ para $26,9 \%$ - o que chama atenção principalmente pe- lo fato da amostra ser relativamente jovem. Por outro lado, observamos diminuição de 17,8\% para $10,7 \%$ no tabagismo. Esses dados demonstram que a amostra estudada, mesmo sendo composta por trabalhadores da saúde, apresenta tendências semelhantes às da população brasileira em geral 31 .

A literatura demonstra que, no Brasil, tabagismo, alimentação não saudável e inatividade física estão entre os principais fatores de risco compartilhados para as doenças crônicas não transmissíveis mais prevalentes. $\mathrm{O}$ aumento na proporção de obesidade que a população brasileira vem sofrendo está associada a mudanças na dieta e nos níveis de atividade física, o que está relacionado ao declínio no gasto energético ocupacional devido ao aumento da força de trabalho no setor de serviços 30 , entre estes os serviços de saúde 7,8; o aumento nos recursos humanos nos serviços de saúde também é observado nos CAPS, já que é um serviço que vem crescendo ano a ano e, segundo o Ministério da Saúde o número de unidades passou de 148 em 1998 para 1.742 em 201114.

Essas transformações podem ter influenciado no aumento da obesidade nas amostras estudadas. Além disso, mesmo com a diminuição no tabagismo, estudo de base populacional realizado no Brasil com sujeitos adultos demonstrou associação entre consumo de tabaco e obesidade abdominal, com risco de obesidade abdominal de $41 \%(\mathrm{p}=0,01)$ para sujeitos inativos fisicamente 36 .

Mesmo com o aumento das doenças crônicas não transmissíveis, observamos diminuição na prevalência de distúrbios psiquiátricos menores de $11 \%$ em 2006 para 8,4\% em 2011, contudo, mulheres foram as mais acometidas $(12,4 \% \mathrm{e}$ $9,2 \%)$ em relação aos homens (6,2\% e 5,5\%), o que pode ser explicado pela maior proporção de mulheres, tanto em 2006 (77,7\%) quanto em 2011 (79,9\%), o que é semelhante a outro trabalho realizado em 2007 com todos os CAPS do Estado do Ceará, que encontrou prevalência de mulheres de $71,8 \%$ 37. Em trabalhadores de UBS da região Sul do Brasil, a prevalência de mulheres variou de $76,0 \%$ a $86 \%$ 15,17. Entretanto, as prevalências de distúrbios psiquiátricos menores observadas em nosso trabalho (11\% e 8,4\%) são inferiores às relatadas na literatura. Estudo de revisão realizado com a população brasileira adulta aponta proporções de $20 \%$ a $56 \%$, sendo que as mulheres são as mais afetadas ${ }^{38}$. Além disso, as prevalências totais encontradas em nosso trabalho também são menores do que as relatadas na literatura por estudos que também utilizaram o SRQ-20 no Sul do Brasil. Estudo de base populacional demonstrou prevalência total de $28,5 \%$; 
as mulheres também foram as mais afetadas e apresentaram $62 \%$ de risco $(\mathrm{p}<0,001)$ em relação aos homens 39 .

Estudo realizado com trabalhadores de UBS apresentou prevalência de $15,6 \%$, com valores muito semelhantes para homens e mulheres 16 , o que difere do nosso estudo que encontrou proporções maiores em mulheres. Pesquisas com trabalhadores de outros setores encontraram em professoras pré-escolares $17,8 \% 4$, em trabalhadores de transporte coletivo $8,6 \%$, sendo $6,6 \%$ em motoristas e $10,4 \%$ em cobradores 5 , e em trabalhadores de academias 7,6\% 6 . Além disso, estudo realizado com trabalhadores de serviços de saúde mental, em outra região do país, encontrou $15,8 \%$ de distúrbios psiquiátricos menores 40.

A literatura tenta explicar a relação existente entre a mulher e o cuidado à loucura nos diferentes períodos históricos, demonstrando que as mulheres, com o desenvolvimento do capitalismo no Brasil, passaram de prestadoras de cuidado informal, dentro das famílias, para trabalhadoras formais e remuneradas no setor da saúde 41,42 .

Todo esse processo coloca em risco a saúde mental dessas mulheres, já que, como trabalhadoras da saúde, também estão submetidas à lógica capitalista de maior produção e mínimo gasto 11. Além disso, a literatura demonstra que trabalhadores de CAPS exercerem suas atividades em um ambiente de disputa entre a burocracia administrativa, demandas dos gestores estadual e municipal, escassez de oferta de serviços de saúde mental, demandas de usuários do serviço, manejo de crise dos usuários. Essa relação gera a fragilidade do trabalhador em decorrência do excesso de exigências, o que pode ser negativo para a sua saúde mental e explicar as maiores prevalências de distúrbios psiquiátricos menores nas mulheres de nosso estudo 43 .

Observamos mudanças na idade, na escolaridade, no tempo de trabalho no CAPS, na formação técnica e na renda das amostras. Além disso, mudança da faixa etária mais prevalente de 30 a 39 anos (30,5\%) em 2006 para 16 a 29 anos (32,7\%) em 2011; diminuição de 62,8\% em 2006 para 57,9\% em 2011, na prevalência de trabalhadores com 13 ou mais anos de estudo; aumento de $29,9 \%$ para $37,8 \%$ de sujeitos com até um ano de trabalho no CAPS; aumento de profissionais de nível técnico de $17,5 \%$ para $20,8 \%$; e aumento de, aproximadamente, $20 \%$ na mediana de renda que passou de $\mathrm{R} \$ 1.050,00$ para R\$ $1.200,00$, sugerindo uma alteração no quadro profissional com a inserção de trabalhadores mais jovens, com formação técnica e com maior renda individual.
O aumento na cobertura de serviços de saúde observado no Brasil também refletiu em nosso estudo 7: 16,9\% da amostra de 2011 eram trabalhadores de CAPS tipo III, este dado não foi coletado em 2006, o que pode ser explicado pelo aumento na cobertura em todo país, mas principalmente na Região Sul que possui a maior cobertura 14. Em 2006 o Brasil contava com 1.010 CAPS e apenas 38 unidades tipo III, em 2011 o número aumentou para 1.742 unidades, sendo 63 tipo III ${ }^{14}$. Na coleta de 2011 o tipo de CAPS apresentou associação positiva com atividade física, tanto na análise bruta quanto na ajustada ( $p=0,02$ ), trabalhadores de CAPS tipo III, ou seja, trabalhadores residentes em municípios de grande porte, com mais de 200 mil habitantes, foram mais ativos fisicamente, com nível de atividade física $83 \%$ maior do que trabalhadores de CAPS tipo I, que são localizados em municípios de pequeno porte.

A literatura tenta identificar e explicar os determinantes da prática de atividade física por diferentes modelos teóricos, um deles é o modelo ecológico ${ }^{44}$, que traz uma visão ampliada de causalidade e coloca a atividade física como um comportamento complexo que sofre influência de fatores psicológicos, biológicos, sociopolíticos e ambientais (individuais, interpessoais e políticas públicas globais, nacionais e regionais) em diferentes fases da vida (infância, adolescência e idade adulta com suas fases de envelhecimento). Entre os fatores ambientais estão os espaços físicos para a prática de atividade física ou o ambiente construído como pistas de caminhadas, ciclovias, segurança na vizinhança que facilite a prática de atividades, e entre os fatores sociopolíticos estão as políticas públicas de urbanização e de promoção da prática de atividade física em nível populacional.

Esses fatores talvez expliquem a associação entre tipo de CAPS e atividade física na amostra de 2011, indicando que municípios de grande porte, que possuem CAPS tipo III, talvez ofereçam condições que facilitem a prática de atividade física, que podem influenciar a motivação dos sujeitos. Além disso, não podemos desconsiderar fatores intrínsecos como a escolha dos sujeitos, os quais podem optar em não praticar atividade física por diferentes fatores. Estudo de base populacional realizado com adultos no Sul do Brasil 45 demonstrou que 73,9\% preferem ter uma dieta saudável como prioridade em saúde, sendo que mulheres referiram dieta saudável com mais frequência do que homens.

Nosso trabalho apresenta limites decorrentes do próprio delineamento (transversal), além do desfecho atividade física ter sido mensurado indiretamente. No entanto, contribui para 
a literatura por apresentar um comparativo entre dois estudos transversais com amostras representativas da população de trabalhadores de CAPS da Região Sul do Brasil, o que garante sua validade externa e lhe atribui características longitudinais.

Em resumo, encontramos baixas prevalências de atividade física em relação à população em geral do Brasil e da Região Sul do país, incluindo trabalhadores de serviços de saúde, o que deve nos deixar atentos em relação a este fator de saúde da população estudada, já que as prevalências relatadas na literatura são de 2 a 5 vezes maiores do que as que encontramos. Houve aumento nas prevalências de obesidade e de doenças crônicas não transmissíveis. Entretanto, observamos aumento da renda, do número de trabalhadores de nível técnico, diminuição do tabagismo e da proporção de distúrbios psiquiátricos menores, sendo que as mulheres ainda são mais acometidas.

\section{Resumen}

El objetivo es presentar las tendencias temporales de actividad física y sus factores asociados en trabajadores de Centros de Atención Psicosocial (CAPS) del sur de Brasil entre 2006 y 2011. Es una investigación transversal, que parte del estudio Evaluación de los CAPS en el sur de Brasil/CAPSUL. Fueron recogidas las variables de salud física, salud mental, a través del Self-Report Questionnaire (SRQ-20), y de actividad física a través del International Physical Activity Questionnaire (IPAQ). Participaron 435 trabajadores en 2006 y 546 trabajadores en 2011. Las prevalencias de actividad física ( $\geq 150$ minutos semanales) fueron un $23,2 \%$ en 2006 y un 17,6\% en 2011 y las de los trastornos psiquiátricos menores un $11 \%$ y un $8,4 \%$, respectivamente. No hubo diferencias en actividad física de hombres y mujeres. En 2006, los sujetos con menor nivel educativo ( $p=$ $0,03)$ y menores ingresos $(p=0,01)$ mostraron un mayor nivel de actividad física. En 2011, los trabajadores de los CAPS, situados en las grandes ciudades, mostraron un mayor nivel de actividad física $(p=0,02)$. Se requieren intervenciones que promuevan la actividad física en esta población, principalmente, en trabajadores de los CAPS residentes en municipios pequeños.

Actividad Motora; Servicios de Salud Mental; Salud Laboral; Salud Mental; Servicios de Salud
Em 2006, sujeitos com menor escolaridade e menor renda apresentaram maiores níveis de atividade física. Em 2011, trabalhadores de CAPS tipo III, localizados em municípios de grande porte, apresentaram maiores níveis de atividade física. Portanto, acreditamos que sejam necessárias intervenções promotoras de atividade física capazes de diminuir a incidência de doenças crônicas não transmissíveis e de obesidade nesta população, principalmente em sujeitos com idades até 49 anos, com maior renda, maior tempo de estudo e que trabalham em CAPS tipo I, localizados em municípios de pequeno porte.

Futuras intervenções e estratégias de ação sobre esses profissionais deverão levar em conta as características ocupacionais desse grupo populacional que, apesar de trabalhar na área da saúde, não está isento dos prejuízos que comportamentos insalubres, como inatividade física, trazem à saúde das populações.

\section{Colaboradores}

J. S. Jerônimo e M. R. Domingues participaram da elaboração do projeto de mestrado que resultou neste artigo, da revisão de literatura, das análises estatísticas e interpretação dos dados, da redação do manuscrito e da aprovação final do mesmo. V. M. R. Jardim participou da concepção do projeto de pesquisa, foi responsável pela confecção dos bancos de dados e disponibilização dos mesmos, participou do tratamento estatístico, da revisão crítica e aprovação final do manuscrito, além de participar das bancas de qualificação do projeto de mestrado e de defesa final da dissertação da qual resultou este artigo. L. P. Kantorski colaborou na concepção do projeto de pesquisa, foi responsável pela confecção dos bancos de dados, contribuiu para a disponibilização dos dados utilizados e participou da revisão crítica e aprovação final do manuscrito.

\section{Agradecimentos}

Ao Ministério da Saúde do Brasil e Capes, pelo apoio financeiro. Ao Programa de Pós-graduação em Educação Física, Escola Superior de Educação, Programa de Pós-graduação em Enfermagem, Faculdade de Enfermagem da Universidade Federal de Pelotas.

\section{Conflito de interesses}

Os autores declaram não haver conflito de interesses. 


\section{Referências}

1. Lee I-M, Shiroma EJ, Lobelo F, Puska P, Blair SN Katzmarzyk PT. Effect of physical inactivity on major non-communicable diseases worldwide: an analysis of burden of disease and life expectancy. Lancet 2012; 380:219-29.

2. Grande AJ, Silva V, Manzatto L, Rocha TBX, Martins GC, Vilela-Junior GB. Comparação de intervenções de promoção à saúde do trabalhador: ensaio clínico controlado randomizado por cluster. Rev Bras Cineantropom Desempenho Hum 2013; 15:27-37.

3. Atkin AJ, Adams E, Bull FC, Biddle SJH. Non-occupational sitting and mental well-being in employed adults. Ann Behav Med 2012; 43:181-8.

4. Silva LG, Silva MC. Condições de trabalho e saúde de professores pré-escolares da rede pública de ensino de Pelotas, RS, Brasil. Ciênc Saúde Coletiva 2013; 18:3137-46.

5. Moura Neto AB, Silva MC. Diagnóstico das condições de trabalho, saúde e indicadores do estilo de vida de trabalhadores do transporte coletivo da cidade de Pelotas - RS. Rev Bras Ativ Fís Saúde 2012; 17:347-58

6. Hartwig TW, Silva MC, Reichert FF, Rombaldi AJ. Condições de saúde de trabalhadores de academias da cidade de Pelotas-RS: um estudo de base populacional. Rev Bras Ativ Fís Saúde 2012; 17:500-11.

7. Paim J, Travassos C, Almeida C, Bahia L, Macinko J. The Brazilian health system: history, advances, and challenges. Lancet 2011; 377:1778-97.

8. Dedecca CS, Trovão CJBM. A força de trabalho no complexo da saúde: vantagens e desafios. Ciênc Saúde Coletiva 2013; 18:1555-67.

9. Tsutsumi A, Kayaba K, Kario K, Ishikawa S. Prospective study on occupational stress and risk of stroke. Arch Intern Med 2009; 169:56-61.

10. Santana VS. Saúde do trabalhador no Brasil: pesquisa na pós-graduação. Rev Saúde Pública 2006; 40:101-11.

11. Bernardo MH, Seligmann-Silva E, Maeno M, Kato M. Ainda sobre a saúde mental do trabalhador. Rev Bras Saúde Ocup 2011; 36:8-11.

12. Ramminger T. Saúde do trabalhador de saúde mental: uma revisão dos estudos brasileiros. Saúde Debate 2008; 32:60-71.

13. Brasil. Lei no 10.216, de 6 de abril de 2001. Dispõe sobre a proteção e os direitos das pessoas portadoras de transtornos mentais e redireciona o modelo em saúde mental. Diário Oficial da União 2001; 9 abr.

14. Coordenação Geral de Saúde Mental, Álcool e Outras Drogas, Departamento de Ações Programáticas Estratégicas, Secretaria de Atenção à Saúde, Ministério da Saúde. Saúde mental em dados - 10. Brasília: Ministério da Saúde; 2012.

15. Tomasi E, Facchini LA, Piccini RX, Thumé E, Silveira DS, Siqueira FV, et al. Perfil sócio-demográfico e epidemiológico dos trabalhadores da atenção básica à saúde nas regiões Sul e Nordeste do Brasil. Cad Saúde Pública 2008; 24 Suppl 1:S193-201.
16. Dilélio AS, Facchini LA, Tomasi E, Silva SM, Thu mé E, Piccini RX, et al. Prevalência de transtornos psiquiátricos menores em trabalhadores da atenção primária à saúde das regiões Sul e Nordeste do Brasil. Cad Saúde Pública 2012; 28:503-14.

17. Siqueira FCV, Nahas MV, Facchini LA, Piccini RX, Tomasi E, Thumé E, et al. Atividade física em profissionais de saúde do Sul e Nordeste do Brasil. Cad Saúde Pública 2009; 25:1917-28.

18. Kantorski LP, Wetzel C, Schwartz E, Jardim VMR, Heck RM, Bielemann VLM, et al. Uma proposta de avaliação quantitativa e qualitativa de serviços de saúde mental: contribuições metodológicas. Saúde Debate 2009; 33:273-82.

19. World Health Organization. Physical status: the use and interpretation of anthropometry. Report of a WHO Expert Consultation. Geneva: World Health Organization; 1995. (WHO Technical Report Series, 854).

20. Ministério da Saúde. Portaria GM/MS no 336, de 19 de fevereiro de 2002. Define e estabelece diretrizes para o funcionamento dos Centros de Atenção Psicossocial. Diário Oficial da União 2002; $20 \mathrm{fev}$

21. Harding TW, Arango MV, Baltazar J, Climent CE, Ibrahim HH, Ladrido-Ignacio L, et al. Mental disorders in primary health care: a study of their frequency and diagnosis in four developing countries. Psychol Med 1980; 10:231-41.

22. Mari JJ, Williams P. A validity study of a psychiatric screening questionnaire (SRQ-20) in primary care in the city of São Paulo. Br J Psychiatry 1986; 148:23-6.

23. Santos KOB, Araújo TM, Oliveira NF. Estrutura fatorial e consistência interna do Self-Reporting Questionnaire (SRQ-20) em população urbana. Cad Saúde Pública 2009; 25:214-22.

24. Gonçalves DM, Stein AT, Kapczinski F. Avaliação de desempenho do Self-Reporting Questionnaire como instrumento de rastreamento psiquiátrico: um estudo comparativo com o Structured Clinical Interview for DSM-IV-TR. Cad Saúde Pública 2008; 24:380-90.

25. Craig CL, Marshall AL, Sjöström M, Bauman AE, Booth ML, Ainsworth BE, et al. International Physical Activity Questionnaire: 12-country reliability and validity. Med Sci Sports Exerc 2003; 35:1381-95.

26. Matsudo S, Araujo T, Matsudo V, Andrade D, Andrade E, Oliveira LC, et al. Questionário Internacional de Atividade Física (IPAQ): estudo de validade e reprodutibilidade no Brasil. Rev Bras Ativ Fís Saúde 2001; 6:5-18.

27. World Health Organization. Global recommendations on physical activity for health. Geneva: World Health Organization; 2010.

28. Barros AJD, Hirakata VN. Alternatives for logistic regression in cross-sectional studies: an empirical comparison of models that directly estimate the prevalence ratio. BMC Med Res Methodol 2003; $3: 21$. 
29. Hallal PC, Andersen LB, Bull FC, Guthold R, Haskell W, Ekelund U. Global physical activity levels: surveillance progress, pitfalls, and prospects. Lancet 2012; 380:247-57.

30. Schmidt MI, Duncan BB, Silva GA, Menezes AM, Monteiro CA, Barreto SM, et al. Chronic non-communicable diseases in Brazil: burden and current challenges. Lancet 2011; 377:1949-61.

31. Departamento de Vigilância de Doenças e Agravos não Transmissíveis e Promoção de Saúde, Secretaria de Vigilância em Saúde, Ministério da Saúde. Vigitel Brasil 2012: vigilância de fatores de risco e proteção para doenças crônicas por inquérito telefônico. Brasília: Ministério da Saúde; 2013.

32. Hallal PC, Matsudo SM, Matsudo VKR, Araújo TL, Andrade DR, Bertoldi AD. Physical activity in adults from two Brazilian areas: similarities and differences. Cad Saúde Pública 2005; 21:573-80.

33. Lopes JA, Longo GZ, Peres KG, Boing AF, Arruda MP. Fatores associados à atividade física insuficiente em adultos: estudo de base populacional no Sul do Brasil. Rev Bras Epidemiol 2010; 13:689-98.

34. Siqueira FV, Facchini LA, Piccini RX, Tomasi E, Thumé E, Silveira DS, et al. Atividade física em adultos e idosos residentes em áreas de abrangência de unidades básicas de saúde de municípios das regiões Sul e Nordeste do Brasil. Cad Saúde Pública 2008; 24:39-54.

35. Siqueira FV, Facchini LA, Silveira DS, Piccini RX, Tomasi E, Hallal PC. Leisure-time physical activity among adult and elderly individuals in Brazil: a countrywide analysis. J Phys Act Health 2011; 8:891-7.

36. Pinho CPS, Diniz AS, Arruda IKG, Batista Filho M, Coelho PC, Sequeira LAS, et al. Prevalência e fatores associados à obesidade abdominal em indivíduos na faixa etária de 25 a 59 anos do Estado de Pernambuco, Brasil. Cad Saúde Pública 2013; 29:313-24.

37. Rigotto RM, Teixeira ACA, Pinheiro CHL, Tófoli LF, Godoy MGC, Cavalcante NC, et al. Análise das condições organizacionais e de seu impacto sobre a saúde dos trabalhadores dos centros de atenção psicossocial do Ceará. Relatório final. http://www. observarh.org.br/observarh/repertorio/Reperto rio_ObservaRH/CETREDE/Analise_condicoes_ org.pdf (acessado em 01/Dez/2013).
38. Santos EG, Siqueira MM. Prevalência dos transtornos mentais na população adulta brasileira: uma revisão sistemática de 1997 a 2009. J Bras Psiquiatr 2010; 59:238-46.

39. Costa JSD, Menezes AMB, Olinto MTA, Gigante DP, Macedo S, Britto MAP, et al. Prevalência de distúrbios psiquiátricos menores na Cidade de Pelotas, RS. Rev Bras Epidemiol 2002; 5:164-73.

40. De-Marco PF, Cítero VA, Moraes E, Nogueira-Martins LA. O impacto do trabalho em saúde mental: transtornos psiquiátricos menores, qualidade de vida e satisfação profissional. J Bras Psiquiatr 2008; 57:178-83.

41. Pegoraro RF, Caldana RHL. Mulheres, loucura e cuidado: a condição da mulher na provisão e demanda por cuidados em saúde mental. Saúde Soc 2008; 17:82-94.

42. Alves AES. Divisão sexual do trabalho: a separação da produção do espaço reprodutivo da família. Trab Educ Saúde 2013; 11:271-89.

43. Ramminger T, Brito JC. "Cada CAPS é um CAPS": uma coanálise dos recursos, meios e normas presentes nas atividades dos trabalhadores de saúde mental. Psicol Soc 2011; 23:150-60.

44. Bauman AE, Reis RS, Sallis JF, Wells JC, Loos RJF, Martin BW. Correlates of physical activity: why are some people physically active and others not? Lancet 2012; 380:258-71.

45. Reichert FF, Domingues MR, Hallal PC, Azevedo MR, Siqueira FV, Barros AJD. Priorities in health: what do they mean to Brazilian adults? Cad Saúde Pública 2010; 26:775-85.

Recebido em 27/Mar/2014

Versão final reapresentada em 15/Jun/2014

Aprovado em 10/Jul/2014 